\title{
Turbulence/outflows perpendicular to low-power jets in Seyfert galaxies $\dagger$
}

\section{Giacomo Venturi $\mathbf{i}^{1,2}\left(\mathbb{D}\right.$, Alessandro Marconi ${ }^{3,2}$, Matilde Mingozzi $^{4}{ }^{4}$, Giovanni Cresci ${ }^{2}$, Stefano Carniani ${ }^{5,2}$ ald and Filippo Mannucci ${ }^{2}$}

\author{
${ }^{1}$ Instituto de Astrofísica, Pontificia Universidad Católica de Chile, \\ Avda. Vicuña Mackenna 4860, 8970117, Macul, Santiago, Chile \\ email: gventuri@astro.puc.cl \\ ${ }^{2}$ INAF - Osservatorio Astrofisico di Arcetri, Largo E. Fermi 5, I-50125, Firenze, Italy \\ ${ }^{3}$ Dipartimento di Fisica e Astronomia, Università degli Studi di Firenze, Via G. Sansone 1, \\ I-50019, Sesto Fiorentino, Firenze, Italy \\ ${ }^{4}$ INAF - Osservatorio Astronomico di Padova, Vicolo dell'Osservatorio 5, 35122, Padova, Italy \\ ${ }^{5}$ Scuola Normale Superiore, Piazza dei Cavalieri 7, I-56126 Pisa, Italy
}

\begin{abstract}
We present recent results from our MAGNUM survey of nearby active galactic nuclei (AGN), which exploits observations from the optical/near-IR integral field spectrograph MUSE at VLT. We detect strongly enhanced line widths in emission line maps of four galaxies perpendicularly to their low-power jets and AGN ionisation cones, indicative of turbulent/outflowing material. The observation of a similar phenomenon in other works suggests that it originates from an interaction mechanism between the jet and the galaxy disc through which it propagates.
\end{abstract}

Keywords. galaxies: jets, galaxies: Seyfert, galaxies: individual (IC 5063, NGC 5643), galaxies: ISM, galaxies: kinematics and dynamics, techniques: spectroscopic

\section{Introduction}

Ionised outflows are routinely observed in active galactic nuclei (AGN), either powered by the strong AGN radiation pressure or by powerful jets (see e.g. Fabian 2012).

Here we focus on new results from our 10-to-100 pc spatially-resolved MAGNUM survey (Measuring Active Galactic Nuclei Under MUSE Microscope) of nearby AGN (e.g. Venturi et al. 2017, 2018; Mingozzi et al. 2019) observed with the optical and nearIR integral field spectrograph MUSE at VLT (Bacon et al. 2010), revealing a peculiar phenomenon in objects hosting low-power jets.

\section{Turbulence/outflows perpendicular to low-power jets}

In Fig. 1 we present MUSE maps of two Seyfert galaxies from our survey, IC 5063 and NGC 5643. Here, while the high bulk velocity gas (panels b and e) indicates an outflow in the direction of the AGN ionisation cones (traced by [O III], panels a and d), as normally expected in AGN (e.g. Fischer et al. 2013), a strongly enhanced line velocity width ( $\gtrsim 800 \mathrm{~km} / \mathrm{s}$; panels $\mathrm{c}$ and $\mathrm{f}$ ), indicating turbulent/outflowing motions with low bulk velocity, is observed instead perpendicularly to the ionisation cones and radio jets (black contours in panels $\mathrm{c}$ and $\mathrm{f}$ ). We detect this phenomenon in two other galaxies of

$\dagger$ Based on observations made with ESO Telescopes at the La Silla Paranal Observatory under program IDs 094.B-0321(A), 60.A-9339(A), 095.B-0532(A).

(C) The Author(s), 2021. Published by Cambridge University Press on behalf of International Astronomical Union. This is an Open Access article, distributed under the terms of the Creative Commons Attribution licence (http://creativecommons.org/licenses/by/4.0/), which permits unrestricted re-use, distribution, and reproduction in any medium, provided the original work is properly cited. 


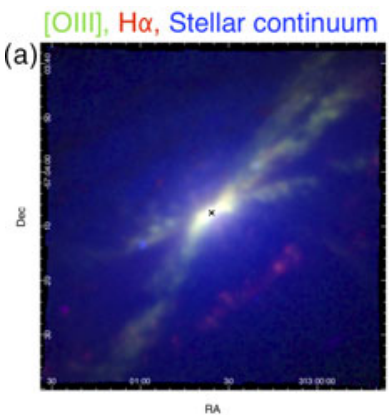

[OIII] flux |200-1000| km/s
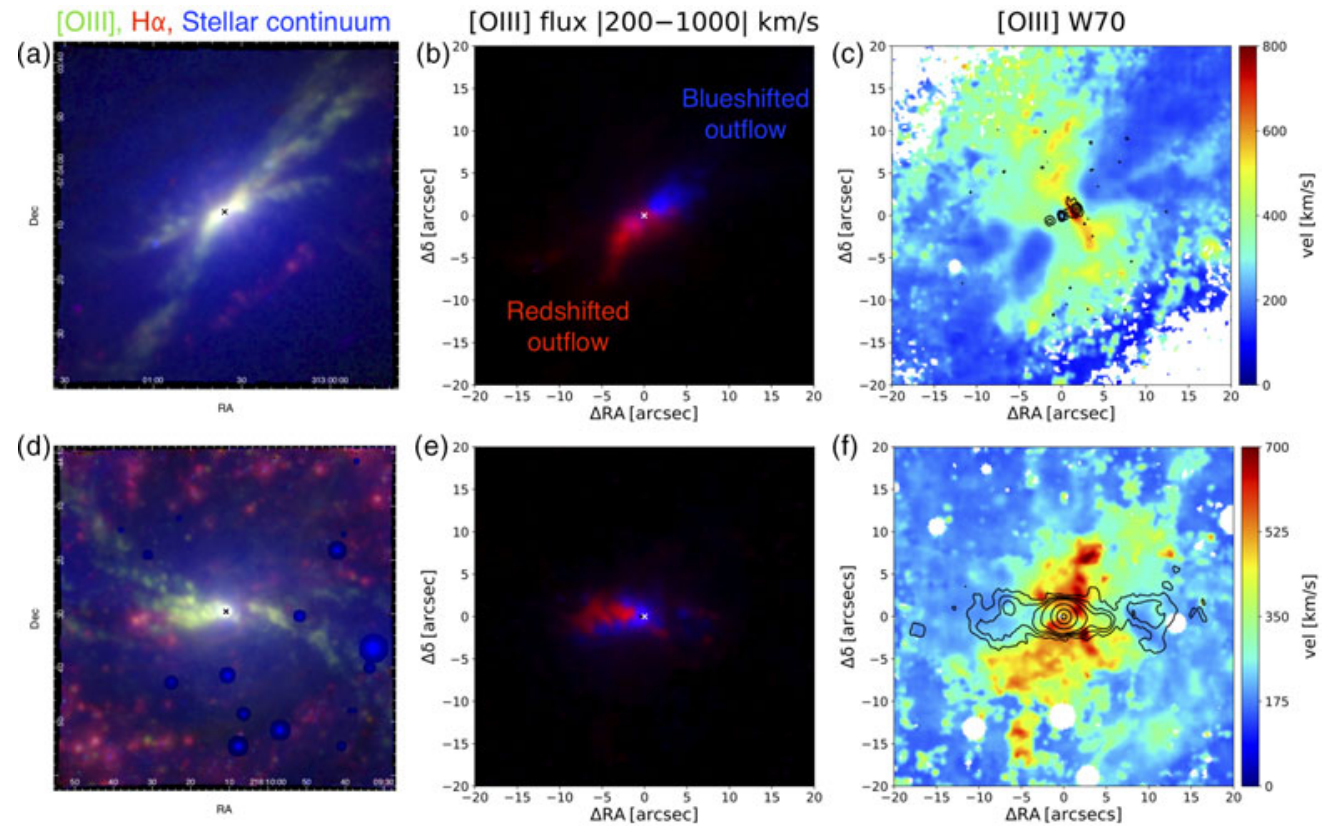

Figure 1. VLT/MUSE maps of IC 5063 (top) and NGC 5643 (bottom). a) and d) Flux maps of ionised gas, [O III] $\lambda 5007$ (green) and $\mathrm{H} \alpha$ (red), and stellar continuum (blue). b) and e) Bulk of the high-velocity outflow, traced by the flux of [O III] line profile integrated in the velocity range $\pm|200-1000| \mathrm{km} / \mathrm{s}$ (blue if approaching, red if receding) with respect to the stellar velocity in each spaxel (to exclude contributions from gas rotating in the disc). c) and f) [O III] W70 line velocity width map (i.e difference between the 85th- and 15th-percentile velocities of the line profile), with radio jet contours superimposed, ATCA $17.8 \mathrm{GHz}$ from Morganti et al. (2007) for IC 5063, 8.4 GHz VLA from Leipski et al. (2006) for NGC 5643.

our sample, NGC 1068 and NGC 1386. We note that all the four mentioned galaxies host a low-power radio jet with low inclinations to galaxy disc.

\section{Discussion and Conclusions}

We have shown MUSE emission-line maps of two nearby Seyfert galaxies from our MAGNUM survey, which - together with two other sources of the sample - exhibit enhanced line velocity widths perpendicularly to their low-power radio jets and ionisation cones, indicative of turbulent/outflowing motions. A similar phenomenon has been reported in other recent works also in galaxies hosting jets lying low onto the galaxy disc (e.g. Shin et al. 2019). This suggests that the jets, through the interaction with the gas in the disc, are responsible for generating the observed perpendicular turbulence/outflow. Moreover, our results confirm that not only powerful jets in "radio loud" objects (e.g. Nesvadba et al. 2008) are capable of affecting the gas in the host, but also low-power jets residing in "radio quiet" galaxies, as indicated by recent works (e.g. Jarvis et al. 2019).

\section{References}

Bacon, R., Accardo, M., Adjali, L., et al. 2010, Proc. SPIE, 7735, 773508

Fabian, A. C. 2012, ARAEAA, 50, 455

Fischer, T. C., Crenshaw, D. M., Kraemer, S. B., et al. 2013, ApJS, 209, 1

Jarvis, M. E., Harrison, C. M., Thomson, A. P., et al. 2019, MNRAS, 485, 2710

Leipski, C., Falcke, H., Bennert, N., et al. 2006, A\&A, 455, 161

Mingozzi, M., Cresci, G., Venturi, G., et al. 2019, A\&A, 622, A146 
Morganti, R., Holt, J., Saripalli, L., et al. 2007, A\&AA, 476, 735

Nesvadba, N. P. H., Lehnert, M. D., De Breuck, C., et al. 2008, A\&\&A, 491, 407

Shin, J., Woo, J.-H., Chung, A., et al. 2019, ApJ, 881, 147

Venturi, G., Marconi, A., Mingozzi, M., et al. 2017, Front. Astron. Space Sci., 4, 46

Venturi, G., Nardini, E., Marconi, A., et al. 2018, A\&EA, 619, A74 